Article

\title{
Facial Mimicry and Social Context Affect Smile Interpretation
}

\author{
Anna Orlowska1, Magdalena Rychlowska², Piotr Szarota ${ }^{3}$, and Eva G. Krumhuber,** \\ 1 Polish Academy of Sciences; anna.barbara.orlowska@gmail.com \\ 2 Queen's University Belfast; m.rychlowska@qub.ac.uk \\ 3 Polish Academy of Sciences; szarota@psychpan.waw.pl \\ 4 University College London; e.krumhuber@ucl.ac.uk \\ * Correspondence: e.krumhuber@ucl.ac.uk;
}

\begin{abstract}
Theoretical accounts and empirical research suggest that people use various sources of information, including sensorimotor simulation and social context, while judging emotional displays. However, the evidence on how those factors can interplay is limited. The present research tested whether social context information has a greater impact on perceivers' smile judgments when mimicry is experimentally restricted. In Study 1, participants watched images of affiliative smiles presented with verbal descriptions of situations associated with happiness or politeness. Half the participants could freely move their faces while rating the extent to which the smiles communicated affiliation, whereas for the other half mimicry was restricted via a pen-in-mouth procedure. As predicted, smiles were perceived as more affiliative when the social context was polite than when it was happy. Importantly, the effect of context information was significantly larger among participants who could not freely mimic the facial expressions. Study 2 replicated these findings, thereby controlling for empathy and mood, and showed that social context also influences smile discrimination. Together, the findings extend the evidence on the role of verbal information in the interpretation of facial expressions and suggest that mimicry importantly modulates the impact of social context information on smile perception.
\end{abstract}

Keywords: mimicry; social context; facial expressions; smile; emotion recognition

\section{Introduction}

The ability to understand other people's emotions and to judge their facial expressions is essential for social interaction [1]. Although prototypical emotion expressions can be easily recognized based on distinct facial patterns, nuanced displays are more difficult to discern due to their subtlety and complexity [2]. Distinguishing between different types of smiles is an example of such complex recognition task. Even though as many as 18 types of smiles have been described in the literature [3], the most commonly used smile typology is the distinction between enjoyment (genuine, Duchenne) and non-enjoyment (posed, non-Duchenne) smiles. Accordingly, smiles that involve a contraction of both the zygomaticus major muscle (Action Unit (AU) 12; Facial Action Coding System (FACS [4]), and orbicularis oculi muscle (AU 6) are considered to be genuine, whereas non-enjoyment smiles lack activation of the latter muscle. Besides this distinction, smiles can be categorized based on their social function (e.g., [5,6]). In particular, Niedenthal and colleagues [6] proposed a classification of smiles related to three main areas of social interaction: reward smiles, which communicate positive affective states; affiliation smiles, which communicate prosocial motives; and dominance smiles, associated with negotiating and maintaining one's social status. Recent studies reveal that these three smile types are morphologically different [7]. Also, people are able to identify and distinguish them [8], suggesting that they are perceptually discernible. 
Despite these differences, judging smiles may still be challenging as they involve complex facial movements $[9,10]$ ) or facial actions co-occurring with the contraction of the zygomaticus major muscle [7]. In addition, the multitude of messages the smiles convey suggest that their interpretation is influenced by the affective state of the perceiver or characteristics of the situation. In other words, although the analysis of the morphology of smiles is necessary to judge these facial expressions, it may not be sufficient for an accurate interpretation of their meaning $[6,11]$. Such inference may be guided by other mechanisms, including sensorimotor simulation $[6,12]$ and contextual influences (e.g., $[13,14])$.

The term "sensorimotor simulation" describes an active representation of the other person's state, generated in the motor, somatosensory, and reward brain circuits $[15,16]$. This complex process is often indexed by facial mimicry [12] or a spontaneous, unconscious imitation of other's facial expressions [17]. Interestingly, the empirical evidence on the role of mimicry in emotion processing has been mixed so far. Moreover, the effects of mimicry can be explained by other variables such as empathy [18] or mood $[19,20]$. Although some studies suggest that disrupting facial movements decreases observers' ability to judge emotion expressions, (e.g., [21,22,23]), others do not show associations between the extent of facial mimicry and the accuracy of emotion recognition $[8,24,25]$. One potential explanation for such discrepancies is that mimicry and sensorimotor processes are especially important in challenging tasks; for example, when observers judge non-prototypical facial expressions [6,11,12]. Interpreting subtle variations between different smile types is an example of such a task, which is likely to be informed by the perceiver's facial mimicry. In line with this notion, studies show that when people are unable to freely move their facial muscles, the ability for inferring other's emotions, in particular expression authenticity, is impaired [21,22,23,26].

Beyond sensorimotor processes, another important factor in smile interpretation is the observer's expectation about what the smile means in a particular situation $[13,27,28]$. Facial expressions do not appear in isolation but are embedded within a given context [29]. As a result, the interpretation of facial expressions is often guided by extraneous factors such as body posture (e.g., [30]), the presence of other people (e.g., [31]), group status or cultural background of the expresser (e.g., [32]). One particularly important source of such influences is the presence of verbal information about the meaning of facial expressions [33]. Psychophysiological research suggests that learned categories and prior knowledge guide the extraction of information from visual objects such as faces [34]. Consistently, facial expressions are easier to recognize when observers are provided with labels that reduce ambiguity and boost categorical perception [33,35,36]. When emotionally ambiguous faces (e.g., blends of anger and sadness) are labelled as "angry", they are further remembered as angrier than the same faces labelled as "sad" [37]. Ambiguous smiles are more likely to be categorized and recalled as genuine when they are paired with contexts implying happiness than when paired with contexts that imply the need for being polite [14,38]. Facial expression processing can also be impaired by providing verbal information that is incongruent with the visual percept $[39,40,41]$. Beyond simply labelling facial expressions, verbal information can also refer to events and situations that observers associate with specific feeling states based on their experiential knowledge [42].

Importantly, the influences of sensorimotor simulation and contextual information on the processing of facial expressions are likely to be non-independent. According to the Accessibility Model of Emotion Self-reports [43], people are more likely to interpret feelings using generalized beliefs and stereotypes when the direct emotion experience is not accessible. Applying this reasoning to facial expression processing, when judging emotion displays such as smiles, observers may use two sources of information: their direct bodily experience resulting from seeing and simulating the expression; and their conceptual knowledge about what this expression means in a given context. However, alterations of facial mimicry, the resulting disruption of sensorimotor simulation, and the 
reduced emotion experience may increase observers' reliance on their conceptual knowledge about the meaning of a given facial expression. Research on emotion expression in groups indirectly supports this prediction. As such, people tend to mimic facial displays of their in-group members to a greater extent than facial displays of members of other groups [44]. Also, the interpretation of facial expressions of out-group members is influenced by stereotypic beliefs about the target group [45]. Surprisingly, there exists only one empirical study to date that provides a direct test of the joint influences of facial mimicry and social context on the interpretation of facial expressions. By asking participants to hold a pencil in their mouth Maringer et al. [21] (Study 2), showed that restricting mimicry increased their reliance on contextual information about the meaning of genuine smiles. Specifically, participants presented with positive contextual information rated smiles as more genuine than those given ambiguous information. Although this research provides an interesting initial demonstration of how both facial mimicry and observers' semantic knowledge about the context contribute to smile interpretation, several limitations should be noted. First, the results are based on small sample sizes, thereby risking type 1 error, an issue particularly pertinent given recent failures of reproducibility in the facial feedback literature [46,47]. Second, the study used computer-generated faces as stimuli, which raises objections regarding the ecological validity and generalization of the findings to real human expressions [48].

\section{The Present Research}

The aim of the present research is to replicate and extend prior findings on how mimicry restriction and verbal information about the social context affect smile interpretation. For this purpose, we conducted two adequately powered studies employing photographs of smiling people as stimuli. Given that posed smiles convey more ambiguous messages and are harder to classify than genuine enjoyment smiles $[7,8,49]$, the study's focus was on non-enjoyment smiles.

In Study 1, participants watched images of smiles presented either in a happy context in which genuine expressions would be expected or in a polite context in which posed expressions would be expected (between-subjects). For half of the participants, facial mimicry was restricted with a procedure similar to the one employed by Maringer and colleagues [21] (see also [20]), such that participants were asked to keep a pencil sidewise in their mouth without exerting any pressure. We predicted that judgments of the extent to which the smiles were affiliative (versus happy) would be strongly influenced by the context in participants whose mimicry was restricted. Such influence should be weaker for participants who could freely mimic the observed smiles.

Study 2 aimed to address a similar question with a different set of real human smiles and manipulated social context as a within-subjects variable. In addition to participants' judgments of the smile meaning, we examined the extent to which participants were able to correctly discriminate smiles that they had previously seen. Using these two dependent measures allowed us to examine the extent to which the effects of context and mimicry restriction vary with task demands [34]. In light of previous research showing that facial mimicry interacts with other variables such as empathy [18,50,51], and exerts effects similar to mood in expression perception [20], we further included empathy and mood as control variables. Similar to Study 1, we predicted that smile judgments would be more strongly influenced by social context when mimicry is inhibited than free.

\section{Study 1. Materials and Methods}

\subsection{Participants and design}

Both studies were approved by the UCL Ethics Committee. A total of 176 participants were recruited face to face at the university campus or via the departmental subject pool. Responses from three participants who correctly guessed the purpose of the study were excluded from analysis, leaving a final sample of 173 participants (59 male, $M_{\text {age }}=21.20$ 
years, $S D=4.76)$. All participants identified themselves as White. Sensitivity analysis using $G^{*}$ Power 3.1 [52] indicated that this sample size was sufficient to detect a mediumsized interaction effect $(f=0.25)$ with $90 \%$ statistical power $(\alpha=0.05)$.

The study manipulated facial mimicry (free vs. restricted) and the context in which smiles were displayed (polite vs. happy) in a between-subjects experimental design. Participants were randomly assigned to one of the four conditions, resulting in approximately 43 persons per group. Ethical approval was granted by the departmental ethics committee. Subjects provided written informed consent prior to participation.

\subsection{Materials}

The facial stimuli consisted of full color, frontal images (size: $960 \times 540$ pixels) of eight (four female) White adult faces with direct gaze and neutral background. Images were retrieved from a stimulus set developed by Martin and colleagues [53] and displayed a non-enjoyment smile corresponding to the description of affiliation smiles [7]. In morphological terms (FACS) [4], the smile expressions involved the Lip Corner Puller (Action Unit (AU) 12) and Chin Raiser (AU17), with or without the Brow Raiser (AU1+2).

We conducted a pilot study to select the verbal context labels for the main experiment. For this, a separate group of participants ( $N=33 ; 27$ women) rated the likely occurrence of a reward, affiliation, and dominance smile [6,7] in 15 different job-related situations. In order to choose situations most conducive to reward (enjoyment) and affiliation (non-enjoyment smiles), we opted for two verbal descriptions that participants most frequently associated with affiliation smiles ("smiling during a job interview", 97\%) and reward smiles ("smiling after receiving a dream job offer", 91\%).

\subsection{Procedure}

Participants were tested individually on computers running Qualtrics, a web-based software (Provo, UT). Upon arrival, they were informed that the study aimed to develop training materials for improving job interview skills and that their task was to rate smiles, as making appropriate facial expressions can contribute to one's success at a job interview. Participants were then randomly assigned to one of the four experimental conditions. Following the procedure of Maringer and colleagues [21], half the participants learned that people's judgments of others' faces are more objective if their own facial movement is restrained. Hence, they would hold a pencil in their mouth while performing the smile evaluation task. The experimenter explained that the pencil should be held sideways, using lips and teeth, without exerting any pressure, and they then demonstrated the correct way of holding it. Single-use disinfection wipes were provided to clean the pencil before using it. The other half of participants who were assigned to the free mimicry condition did not receive such instructions and were able to freely mimic the smile expressions.

For the manipulation of social context, half the participants were informed that the images they would see featured people who were "smiling during a job interview" (polite condition). The other half was told that the persons were "smiling after receiving a job offer" (happy condition). Prior to the smile evaluation task, we tested whether participants believed that a smile displayed in the polite context would communicate more affiliation and less happiness than a smile displayed in the happy context. To that end, subjects were asked to imagine a person smiling "during a job interview" or "after receiving a job offer", consistent with the condition to which they were assigned. They then rated the extent to which the imagined smile would communicate that the person wanted "to be nice and to express positive intentions" (affiliation rating) or was "feeling happy and content" (happiness rating), using scales ranging from $0 \%$ to $100 \%$. Participants' ratings of these two dimensions were non-independent and had to add up to $100 \%$.

The main smile evaluation task involved the presentation of eight smile expressions in combination with one of the two social context scenarios (happy or polite). The context 
information was shown at the top of the screen together with each stimulus. Presentation order was randomized, with each smile expression being displayed for 3 seconds. For each face-context pair, participants rated the extent to which the smile would communicate that the person wanted "to be nice and to express positive intentions" (ratings of affiliation, from $0 \%$ to $100 \%$ ) or was "feeling happy and content" (ratings of happiness, from $0 \%$ to $100 \%$ ). Again, both ratings were complementary and added up to $100 \%$. At the end of the procedure, participants were probed for suspicion, thanked and debriefed.

\section{Study 1. Results}

\subsection{A priori beliefs}

To examine participants' a priori beliefs about the type of smile expected in the two social context scenarios, we conducted a 2 (context: polite, happy) by 2 (mimicry: free, restricted) between-subjects ANOVA on ratings of affiliation (averaged across the stimulus faces) as the dependent variable. Consistent with predictions, participants expected smiles paired with the polite context to communicate more affiliation ( $M=$ $73.55, S D=16.81$ ), and thus less happiness than smiles paired with the happy context ( $M$ $=29.08, S D=24.31), F(1,169)=194.31, p<.001, \mid \mathrm{p}^{2}=.53$. Participants in the free mimicry condition expected smiles to be less affiliative $(M=46.90, S D=30.41)$ than those in the muscle-restricted condition $(M=56.05, S D=30.08)$, but the main effect of mimicry did not reach conventional levels of significance, $F(1,169)=4.04, p=.05$. The interaction between mimicry and context was not significant, $F(1,169)=0.19, p=.660, \mid \mathrm{p}^{2}=.001$.

\subsection{Smile evaluation}

Ratings of affiliation were averaged across the stimulus faces and analyzed in a between-subjects ANOVA as a function of context and mimicry. The main effect of context was significant, $F(1,169)=55.58, p<.001, \mid \mathrm{p}^{2}=.25$, such that smiles were perceived as more affiliative when they were presented together with a polite $(M=67.42$, $S D=13.92)$ than with a happy context $(M=49.28, S D=18.14)$. Mimicry had no effect on perceived affiliation, $F(1,169)=0.27, p=.606, \mid \mathrm{p}^{2}=.002$. However, there was a significant interaction between context and mimicry, $F(1,169)=7.60, p<.006,\left.\right|_{\mathrm{p}^{2}}=.04$ (see Figure 1). As expected, the impact of context on smile ratings was stronger when facial mimicry was restricted (polite context: $M=71.17, S D=2.35$; happy context: $M=46.38, S D=2.52$, $\left.F(1,169)=51.81, p<.001, \mid \mathrm{p}^{2}=.24\right)$ than free (polite context: $M=63.22, S D=2.49$; happy context: $\left.M=51.81, S D=2.35, F(1,169)=11.11, p=.001, \mid \mathrm{p}^{2}=.06\right)$. 


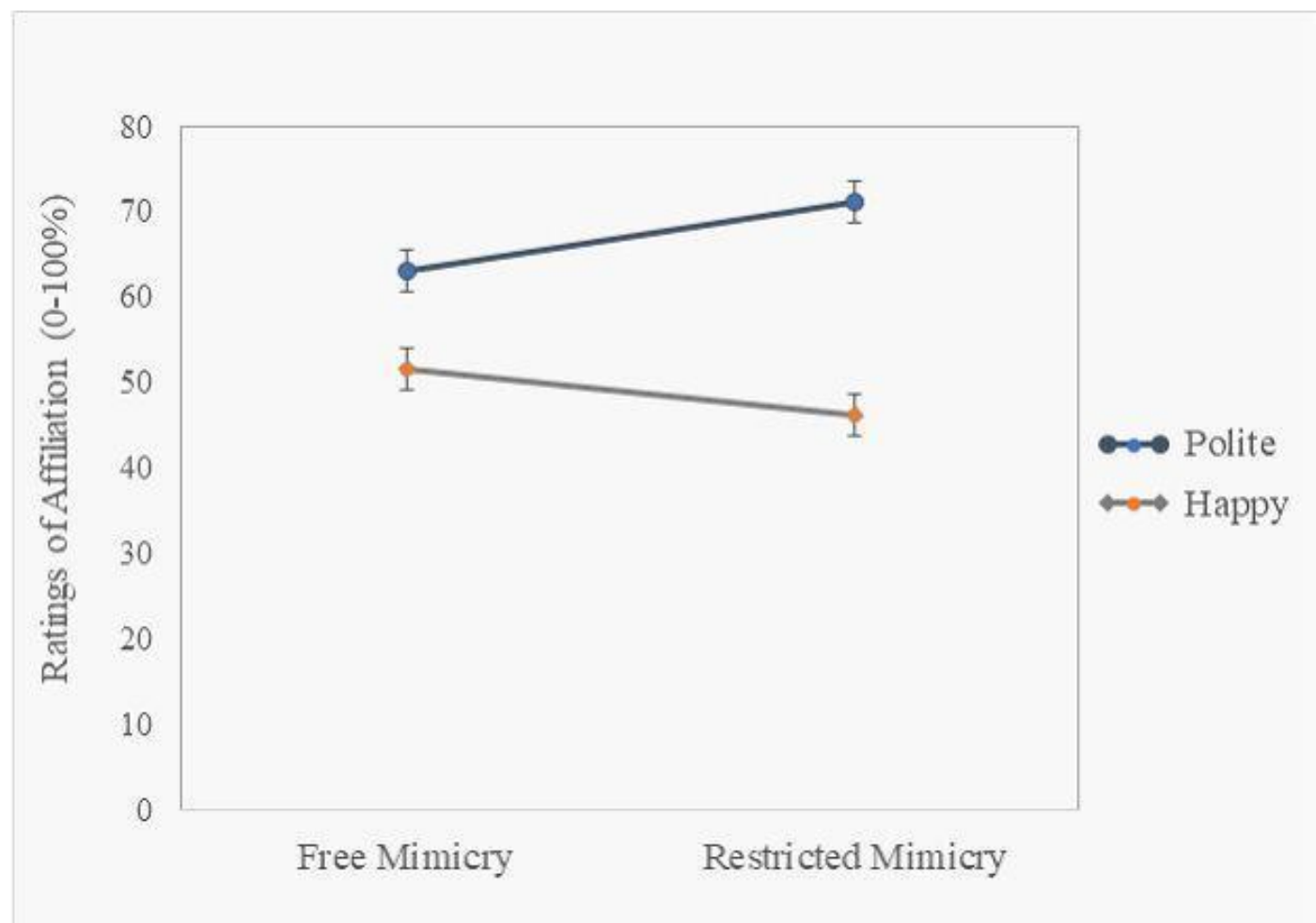

Figure 1. Ratings of affiliation as a function of social context and mimicry in Study 1. Error bars represent standard errors of the mean (SEM).

\section{Study 1. Discussion}

In Study 1, we tested whether social context, as well as the ability to mimic, influence participants' judgments of affiliation smiles. Consistent with our predictions, participants held specific beliefs about the meaning of smiles. Specifically, they believed that smiles displayed during a job interview (polite context) would convey more affiliation than smiles shown after receiving a job offer (happy context). Furthermore, context information affected participants' evaluation of the expression. Specifically, smiles were judged as conveying more niceness and positive intentions and less happiness and contentment when they occurred in the context of a polite than happy situation. Importantly, such contextual influence was found to be stronger for participants whose mimicry was restrained, suggesting that they relied more on conceptual knowledge in order to judge the genuineness of the smile. In Study 2, we aimed to replicate the present findings and control for two potential moderating variables-empathy and mood.

\section{Study 2. Materials and Methods}

The procedure for this study was similar to the one used in Study 1. Participants judged smile expressions presented in a polite and happy context under conditions of free and restricted mimicry. This time, however, we used a different set of facial stimuli. We also manipulated the social context as a within-subjects variable to account for possible individual differences. As in Study 1, participants rated the extent to which each smile communicated affiliation and happiness. In addition to this main task, we assessed participants' ability to discriminate between different types of smiles, which is also likely to be affected by both verbal information [41] and mimicry restriction [54]. Furthermore, we included control measures of empathy and mood. Similar to the first study, we predicted that judgments of smile authenticity would be more strongly influenced by social context when input from mimicry is inhibited rather than free. 


\subsection{Participants and design}

A total of 66 participants ( 50 women, $M_{a g e}=22.71$ years, $S D=5.35$ ) were recruited at the university campus and completed the study without remuneration. Sixty-five participants identified themselves as White and one reported "other ethnical background" (Turkish nationality). Sensitivity analysis using G*Power indicated that this sample size was sufficient to detect a medium-sized interaction effect $(f=0.24)$ with $90 \%$ statistical power $\left(\alpha=0.05, r_{\text {cor }}=0.3\right)$.

The study used a mixed experimental design, with facial mimicry (free vs. restricted) as a between-subjects variable and context information (polite vs. happy) as a within-subjects variable. Participants were randomly assigned to one of the two mimicry conditions (free: 34 participants, restricted: 32 participants). Ethical approval was granted by the departmental ethics committee, and subjects provided written informed consent prior to participation.

\subsection{Materials}

The facial stimuli consisted of full colour, frontal images of 12 White female faces displaying a posed smile and a genuine smile in a total of 24 photographs. All models were presented with direct gaze and on a neutral background. Images were retrieved from stimuli sets developed by Johnston, Miles and Macrae [49] and McLellan, Johnston, Dalrymple-Alford and Porter [55]. All genuine smiles corresponded to the description of a Duchenne smile [56] in that they involved both the Lip Corner Puller (AU12) and Cheek Raiser (AU6). In contrast, posed smiles did not involve AU6.

To measure participants' empathy levels, we used the Empathy Quotient questionnaire (EQ40) [57], which consists of 40 items presented as statements such as "I find it easy to put myself in somebody else's shoes" or "I am good at predicting how someone will feel." Participants have to use a 4-point Likert scale to rate how strongly they agree with the statements. Scores for each item range from 0 to 2 points, and the maximum overall score is 80 , with higher values indicating higher levels of empathy. For the current sample, internal consistency was good at $\alpha=.856$.

\subsection{Procedure}

Participants were tested individually on computers running the Qualtrics survey software. Upon arrival, they were informed that the aim of the study was to develop educational materials for job candidates wanting to improve their job interview skills. They were then provided with brief definitions of each smile type (genuine: "a smile displayed when someone is happy or amused and is truly feeling the emotion"; posed: "a smile that is intentional in the sense that someone wants to be nice and express positive intentions but does not feel the respective emotion").

Prior to the smile evaluation task, we tested participants' beliefs about the meaning of smiles displayed in the polite and happy contexts. Subjects were asked to imagine a person smiling during a job interview and after receiving a job offer, and to rate the extent to which the imagined smile would communicate that the person wanted "to be nice and to express positive intentions" (affiliation rating) or was "feeling happy and content" (happiness rating), using scales ranging from $0 \%$ to $100 \%$. As in Study 1, participants' responses across the two rating dimensions had to add up to $100 \%$. After rating their beliefs about the meaning of smiles displayed in the polite and happy contexts, participants were randomly assigned to one of the two mimicry conditions. The restriction procedure was identical to the one used in Study 1.

The smile evaluation task involved the presentation of the 12 posed smiles (size: $360 \times 480$ pixels) in two blocks (each involving six photographs). The social context information was shown at the top of the screen together with each smile stimulus. To mirror the time course of the two scenarios, the first block always started with a 
description of the polite context ("This person is smiling during a job interview"), followed by the happy context description in the second block ("This person is smiling after receiving a job offer"). The selection of images in each block was counterbalanced across participants. Also, we randomized presentation order within blocks, with every smile expression being displayed for 3 seconds. For each face-context pair, participants rated the extent to which the smile would communicate that the person wanted "to be nice and to express positive intentions" (ratings of affiliation, from $0 \%$ to $100 \%$ ) or was "feeling happy and content" (ratings of happiness, from $0 \%$ to $100 \%$ ). Again, both ratings were complementary and added up to $100 \%$.

After completing the smile evaluation part, participants in the mimicry-restricted condition removed the pencil from their mouth. All participants then rated their mood on a scale ranging from 1 ("very negative") to 7 ("very positive) and completed the EQ questionnaire [57]. Both tasks lasted approximately 5 minutes.

Next, participants engaged in an unexpected two-alternative forced-choice smile discrimination task. On each trial, they saw a posed smile from the first part of the experiment alongside a genuine smile (distractor stimulus) displayed by the same actor (sizes: $250 \times 310$ pixels). The relevant context information, which was the same as shown earlier with the posed smile, was provided with each image pair and presented at the bottom of the screen. The participants' task was to indicate which of the two facial stimuli they had seen earlier. Thus, posed smiles were always the correct response, whereas genuine smiles were foils. Both the target and distractor image remained on the screen until participants submitted their response. We randomized the order of trials and counterbalanced the sides on which each type of smile appeared. Finally, participants were thanked and debriefed. 


\section{Study 2. Results}

\subsection{A priori beliefs}

As in Study 1, we examined participants' a priori beliefs about the type of smile expected in the two social context scenarios. A 2 (context: polite, happy) by 2 (mimicry: free, restricted) mixed ANOVA showed that, consistent with predictions, participants expected smiles paired with the polite context to communicate more affiliation ( $\mathrm{M}=$ $76.48, \mathrm{SD}=17.84)$ than smiles paired with the happy context $(\mathrm{M}=21.41, \mathrm{SD}=20.99), \mathrm{F}(1$, $64)=283.95, \mathrm{p}<.001, \mid \mathrm{p}^{2}=.82$. The main effect of mimicry and the interaction between mimicry and context were not significant, $F(1,64)<0.001, p=.990, \mid \mathrm{p}^{2}<.001$ and $F(1,64)$ $=3.14, \mathrm{p}=.081, \mid \mathrm{p}^{2}=.05$, respectively. Adding mood and empathy as covariates in an ANCOVA did not change the pattern of statistical results and revealed no main effects of or interactions with the covariates ( Fs $<3.42$, ps $>.068$ ).

\subsection{Smile evaluation}

Ratings of affiliation were averaged across the stimulus faces and analyzed in a mixed ANOVA as a function of context and mimicry. The results revealed a main effect of context, $\mathrm{F}(1,64)=98.20, \mathrm{p}<.001, \mid \mathrm{p}^{2}=.60$, such that smiles were perceived as more affiliative when they appeared together with a polite $(M=67.72, S D=11.40)$ than with a happy context $(M=44.60, S D=13.15)$. The main effect of mimicry on perceived affiliation did not reach significance, $\mathrm{F}(1,64)=3.74, \mathrm{p}=.06, \mid \mathrm{p}^{2}=.05$. However, there was a significant interaction between context and mimicry, $\mathrm{F}(1,64)=5.29, \mathrm{p}=.025, \mathrm{p}^{2}=.08$ (see Figure 2). As expected, the impact of social context on smile ratings was stronger when facial mimicry was restricted (polite context: $\mathrm{M}=72.75, \mathrm{SD}=11.40$; happy context: $\left.\mathrm{M}=44.05, \mathrm{SD}=12.02, \mathrm{~F}(1,64)=72.35, \mathrm{p}<.001, \mid \mathrm{p}^{2}=.53\right)$ than free (polite context: $\mathrm{M}=$ 63.00, $\mathrm{SD}=14.58$; happy context: $\left.\mathrm{M}=45.12, \mathrm{SD}=14.30, \mathrm{~F}(1,64)=29.86, \mathrm{p}<.001, \mid \mathrm{p}^{2}=.32\right)$.

When controlling for mood and empathy as covariates in an ANCOVA, the main effect of context, $\mathrm{F}(1,62)=6.77, \mathrm{p}=.012, \mid \mathrm{p}^{2}=.10$, as well as the context by mimicry interaction, $\mathrm{F}(1,62)=4.70, \mathrm{p}=.034, \mathrm{p}^{2}=.07$, remained significant. There were no main effects of or interactions with the covariates (Fs $<1.43$, ps $>.235$ ). 


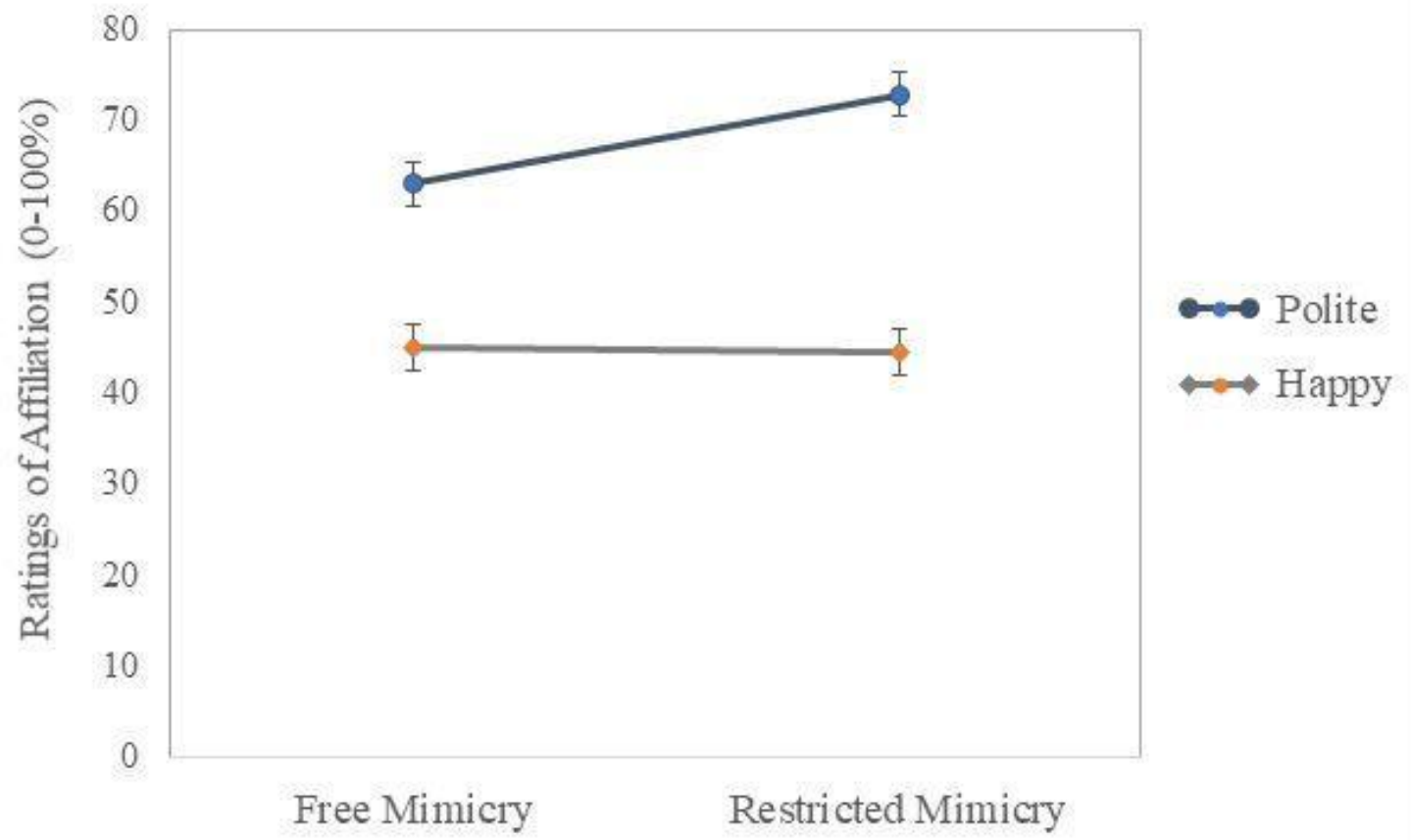

Figure 2. Ratings of affiliation as a function of social context and mimicry in Study 2. Error bars represent SEM.

\subsection{Smile discrimination task}

Percentages of correctly identified posed smiles were averaged across the stimulus faces and analyzed in a mixed ANOVA as a function of context and mimicry. The main effect of context was significant, $\mathrm{F}(1,64)=5.18, \mathrm{p}=.026, \mid \mathrm{p}^{2}=.08$, such that a higher proportion of smiles was correctly identified as posed when they were presented together with a polite $(\mathrm{M}=85.35, \mathrm{SD}=18.83)$ than with a happy context $(\mathrm{M}=77.27, \mathrm{SD}=$ 26.98). Mimicry had no effect on smile recall, $\mathrm{F}(1,64)=0.35, \mathrm{p}=.555, \mid \mathrm{p}^{2}<.01$, and the interaction between context and mimicry was not significant, $\mathrm{F}(1,64)=0.05, \mathrm{p}=.297, \mathrm{p}^{2}$ $=.02$.

An ANCOVA revealed no main effects of or interactions with mood and empathy as covariates ( $\mathrm{Fs}<1.40$, ps $>.263$ ). However, the main effect of context was no longer significant, $\mathrm{F}(1,62)=1.39, \mathrm{p}=.243, \mid \mathrm{p}^{2}=.02$. 


\section{Study 2. Discussion}

In Study 2, we investigated whether social context information and the ability to mimic influence participants' judgments of posed smiles and discrimination of these facial expressions. The findings replicate and extend the results of Study 1. First, participants held specific beliefs about the meaning of smiles produced in a polite and happy context, and thought that the former were likely to communicate more affiliation. Furthermore, providing participants with a label describing a social situation affected the way they interpreted the posed smiles. Specifically, these expressions were perceived as conveying more niceness and positive intentions and less happiness and contentment when they were paired with a polite rather than happy situation. This influence of social context on smile judgments was stronger for participants whose mimicry was restrained, suggesting that they relied more on their conceptual knowledge. None of the above effects was modulated by empathy and mood, which points toward the critical role of mimicry beyond internal factors. Importantly, restricting facial mimicry did not worsen smile discrimination, suggesting that it is unlikely that mimicry inhibition distracted participants from paying attention to the task. Interestingly, social context influenced participants' ability to discriminate smiles such that a polite situation made participants select more posed smiles in comparison with a happy situation. Although this result indicates that context information can affect the way information is retrieved, the effect became non-significant when controlling for empathy and mood in the analysis.

\section{General Discussion}

The present research examined the role of facial mimicry and social context information in the interpretation of non-enjoyment smiles. We tested the influence of these factors in two studies in which we altered participants' facial activity using a standard pen-in-mouth manipulation and exposed them to employment-related descriptions of situations depicting happy and polite context. Participants judged the extent to which the smiles conveyed affiliation versus happiness and discriminated posed from genuine smiles. As predicted, our analyses revealed that in both studies participants perceived smiles as more affiliative when they were presented in a context associated with politeness than in a context judged as happy. In addition, context influenced smile discrimination, such that participants were more likely to select posed rather than genuine smiles when the task was accompanied by the polite context information. These results are in line with previous research documenting the importance of verbal information on the processing of facial expressions [33,37]. Our findings also confirm that people have specific beliefs about the types of smile appropriate in specific social situations and that this conceptual knowledge can shape the way they interpret and perceive smiles.

Furthermore, consistent with existing literature on sensorimotor simulation (e.g., $[6,12]$, the two studies show that experimentally restricting facial mimicry can increase participants' reliance on contextual information. This result is in line with the studies of Maringer and colleagues [21], which, to our knowledge, were the only evidence of joint effects of sensorimotor simulation and social context on smile processing. Here, we replicate and extend these findings using more naturalistic stimuli presenting human actors rather than animated characters. In addition, both studies are confirmatory and adequately powered, which is particularly needed given the recent evidence on low reproducibility of research on facial feedback effects [46].

It is worth noting that, when exploring the discrimination between reward, affiliation, and reward smiles, [8] did not find a significant effect of restricting 
participants' facial movements. One potential explanation of these seemingly inconsistent findings is that the present studies assessed the perceived meaning of a smile within a given situation, whereas in the study by Orlowska and colleagues [8], participants were simply asked to categorize perceived expressions as instances of reward, affiliation, or dominance smiles. It is possible that facial mimicry, as well as other sensorimotor processes, may not be necessary to properly classify the type of smile but play an important role in more challenging tasks, such as interpreting the meaning of a smile in a social situation.

It is also worth highlighting that Maringer et al. [21] (Study 2) did not observe a significant main effect of context. This could be due to the relatively low statistical power of this prior study, involving only 12 participants per condition. Another plausible explanation is the type of smile used. In the present studies, participants were asked to evaluate the meaning of non-enjoyment smiles, whereas genuine smiles served as target stimuli in the study by Maringer and colleagues [21]. As affiliation smiles are more ambiguous and challenging to classify than enjoyment smiles $[7,8]$, we believe that their judgment is more susceptible to the influence of contextual information. Such a tendency may be particularly marked for static stimuli which are more difficult to discern than dynamic expressions [58].

Both studies revealed consistent effects of context information on smile processing. In addition to showing that such information affects smile judgments, Study 2 reveals that verbal labels describing social situation may also affect participants' ability to discriminate smiles. Specifically, compared with a happy situation, a polite situation made participants more likely to select posed smiles. Such results are in line with previous research [41], which suggests that memorization of positive facial expressions is particularly vulnerable to verbal contextual information. Indeed, accurate memory for facial features presumably informs face perception [59], but it has been shown that both face perception and memory are malleable and subject to biases [60]. For instance, social information about a target face can induce assimilation of the face to a categorical prototype, which influences how a face is perceived and remembered [38,61,62]. Given that emotion expressions are often represented as categorical prototypes (i.e. prototypical emotion terms like "anger" or "fear" reflect cognitive representations in memory and language; [63], it is reasonable to expect that such assimilation processes also occur in the case of social context for facial displays. In other words, emotion expressions may be misremembered as having occurred in a social context more prototypical of the category (in this case: smile type).

We did not observe any effects of mimicry restriction in the smile discrimination task. This finding suggests that verbal information affects smile processing to a greater extent than sensorimotor processes. In addition, correct recollection of smiles, as required in the discrimination task, may be less sensitive to disruptions of sensorimotor processing than online judgments of these facial expressions. This result is in line with the findings of the study by [64], who showed that facial mimicry is reduced by inconsistent verbal information in the initial stages of encoding an expression but not when the same expression is viewed later.

Unlike the study of Maringer and colleagues [21], the present experiments controlled for a number of potential confounds. Specifically, Study 2 included measures of participants' empathy levels and mood and accounted for their joint effects [20,50,65]. The effects of restricting facial mimicry on participants' smile judgments remained similar after controlling for these internal factors, suggesting that our findings cannot be explained by participants' lower mood or empathy levels. However, the observed effect of context on discriminating enjoyment from non-enjoyment smiles was no longer 
significant after adding empathy and mood as covariates. Thus, the influence of verbal information may vary depending on task demands and be less marked in discriminating rather than interpreting the meaning of facial expressions. Importantly, we did not find main effects of mimicry on participants' ratings performance in any of the two studies, making it unlikely that inhibiting facial movements distracted participants from paying attention to the task. Such an interpretation was also discarded by the study of Rychlowska and colleagues [26], who showed that judgments of smile genuineness can be disrupted by restricting mimicry but not by other distracting tasks (i.e., holding a squeeze ball or wearing a finger-cuff heart rate monitor).

To our knowledge, our studies are the first to experimentally test the joint influences of social context and restricting facial mimicry on the processing of smiles displayed by human actors. Our findings reveal that people hold specific beliefs about the likelihood of observing non-enjoyment and enjoyment smiles in different social contexts and that this knowledge is used to guide the interpretation and discrimination of smiles. Importantly, this influence is particularly strong when participants' facial mimicry is inhibited by holding a pen in the mouth. The present results have important implications for everyday life, offering insights into mechanisms guiding facial expression processing in clinical populations, such as patients with facial palsy or persons whose facial mimicry is altered due to reduced social motivation [44]. By investigating the interplay of social context and sensorimotor processes, the present research depicts an important first step in this direction and provides promising avenues for future research.

\section{References}

1. Kappas, A.; Krumhuber, E.; Küster, D. Facial behavior. In Nonverbal Communication, Hall J.A.; Knapp M.L., Eds.; De Gruyter Mouton: Berlin, 2013, Volume 2, pp. 131-165. doi: 10.1515/9783110238150.131

2. Hess, U.; Blairy, S.; Kleck, R.E. The intensity of emotional facial expressions and decoding accuracy. J. Nonverbal Behav. 1997, 21, 241-257. doi: 10.1023/A:1024952730333

3. Ekman, P. Telling Lies: Clues to Deceit in the Marketplace, Politics, and Marriage (revised Edition); WW Norton \& Company, 2009.

4. Ekman, P.; Friesen, W. V.; Hager, J. C. Facial Action Coding System: The manual on CD ROM; UT: Research Nexus, 2002.

5. Ambadar, Z.; Cohn, J.F.; Reed, L.I. All smiles are not created equal: morphology and timing of smiles perceived as amused, polite, and embarrassed/nervous. J. Nonverbal Behav. 2009, 33, 17-34. doi: 10.1007/s10919-008-0059-5

6. Niedenthal, P.M.; Mermillod, M.; Maringer, M.; Hess, U. The Simulation of Smiles (SIMS) Model: embodied simulation and the meaning of facial expression. Behav. Brain Sci. 2010, 33, 417-433. doi: 10.1017/S0140525X10000865

7. Rychlowska, M.; Jack, R.E.; Garrod, O.G.B.; Schyns, P.G.; Martin, J.D.; Niedenthal, P.M. Functional smiles: tools for love, sympathy, and war. Psychol. Sci. 2017, 28, 12591270. doi: $10.1177 / 0956797617706082$

8. Orlowska, A.B.; Krumhuber, E.G.; Rychlowska, M.; Szarota, P. Dynamics matter: recognition of reward, affiliative, and dominance smiles from dynamic vs. static displays. Front. Psychol. 2018, 9, 938. doi: 10.3389/fpsyg.2018.00938

9. Krumhuber, E.; Kappas, A. Moving smiles: the role of dynamic components for the perception of the genuineness of smiles. J. Nonverbal Behav. 2005, 29, 3-24. doi: 10.1007/s10919-004-0887-x

10. Krumhuber, E.G.; Manstead, A.S.R. Can Duchenne smiles be feigned? New evidence on felt and false smiles. Emotion 2009, 9, 807-820. doi: 10.1037/a0017844 
11. Hess, U.; Fischer, A. Emotional mimicry as social regulation. Pers. Soc. Psychol. Rev. 2013, 17, 142-157. doi: 10.1177/1088868312472607

12. Wood, A.; Rychlowska, M.; Korb, S.; Niedenthal, P. Fashioning the face: sensorimotor simulation contributes to facial expression recognition. Trends Cogn. Sci. 2016, 20, 227240. doi: 10.1016/j.tics.2015.12.010

13. Hess, U.; Hareli, S. The role of social context for the interpretation of emotional facial expressions. In Understanding Facial Expressions in Communication: Cross-cultural and Multidisciplinary Perspectives; Mandal, M.K., Awasthi, A., Eds.; Springer India: New Delhi, 2015; pp. 119-141. doi: 10.1007/978-81-322-1934-7_7

14. Namba, S.; Rychlowska, M.; Orlowska, A.; Aviezer, H.; Krumhuber, E.G. Social context and culture influence judgments of non-Duchenne smiles. Journal of Cultural Cognitive Science 2020, 4, 309-321. doi: 10.1007/s41809-020-00066-1

15. Ferrari, P.F.; Coudé, G. Mirror neurons, embodied emotions, and empathy. Neuronal Correlates of Empathy 2018, 67-77. doi: 10.1016/B978-0-12-805397-3.00006-1

16. Pitcher, D.; Garrido, L.; Walsh, V.; Duchaine, B.C. Transcranial magnetic stimulation disrupts the perception and embodiment of facial expressions. J. Neurosci. 2008, 28, 89298933. doi: 10.1523/JNEUROSCI.1450-08.2008

17. Dimberg, U.; Thunberg, M. Rapid facial reactions to emotional facial expressions. Scand. J. Psychol. 1998, 39, 39-45. doi: 10.1111/1467-9450.00054

18. Kosonogov, V.; Titova, A.; Vorobyeva, E. Empathy, but not mimicry restriction, influences the recognition of change in emotional facial expressions. Q. J. Exp. Psychol. 2015, 68, 2106-2115. doi: 10.1080/17470218.2015.1009476

19. Forgas, J.P.; East, R. How real is that smile? Mood effects on accepting or rejecting the veracity of emotional facial expressions. J. Nonverbal Behav. 2008, 32, 157-170. doi: 10.1007/s10919-008-0050-1

20. Niedenthal, P.M.; Brauer, M.; Halberstadt, J.B.; Innes-Ker, Å.H. When did her smile drop? Facial mimicry and the influences of emotional state on the detection of change in emotional expression. Cognition Emotion 2001, 15, 853-864. doi:

10.1080/02699930143000194

21. Maringer, M.; Krumhuber, E.G.; Fischer, A.H.; Niedenthal, P.M. Beyond smile dynamics: mimicry and beliefs in judgments of smiles. Emotion 2011, 11, 181-187. doi: $10.1037 / \mathrm{a} 0022596$

22. Oberman, L.M.; Winkielman, P.; Ramachandran, V.S. Face to Face: blocking facial mimicry can selectively impair recognition of emotional expressions. Soc. Neurosci. 2007, 2, 167-178. doi: 10.1080/17470910701391943

23. Ponari, M.; Conson, M.; D’Amico, N.P.; Grossi, D.; Trojano, L. Mapping correspondence between facial mimicry and emotion recognition in healthy subjects. Emotion 2012, 12, 1398-1403. doi: 10.1037/a0028588

24. Blairy, S.; Herrera, P.; Hess, U. Mimicry and the judgment of emotional facial expressions. J. Nonverbal Behav. 1999, 23, 5-41. doi: 10.1023/A:1021370825283

25. Korb, S.; With, S.; Niedenthal, P.; Kaiser, S.; Grandjean, D. The perception and mimicry of facial movements predict judgments of smile authenticity. PLoS One 2014, 9 , e99194. doi: 10.1371/journal.pone.0099194

26. Rychlowska, M.; Cañadas, E.; Wood, A.; Krumhuber, E.G.; Fischer, A.; Niedenthal, P.M. Blocking mimicry makes true and false smiles look the same. PLoS One 2014, 9, e90876. doi: 10.1371/journal.pone.0099194

27. Fischer, A.; Hess, U. Mimicking emotions. Curr Opin Psychol 2017, 17, 151-155. doi: 10.1016/j.copsyc.2017.07.008

28. Greenaway, K.H.; Kalokerinos, E.K.; Williams, L.A. Context is everything (in emotion research). Soc. Personal. Psychol. Compass 2018, 12, e12393. doi: 10.1111/spc3.12393 
29. Barrett, L.F.; Mesquita, B.; Gendron, M. Context in emotion perception. Curr. Dir. Psychol. Sci. 2011, 20, 286-290. doi: 10.1177/0963721411422522

30. Aviezer, H.; Hassin, R.R.; Ryan, J.; Grady, C.; Susskind, J.; Anderson, A.; Moscovitch, M.; Bentin, S. Angry, disgusted, or afraid? Psychol. Sci. 2008, 19, 724-732. doi: 10.1111/j.1467-9280.2008.02148.x

31. Gray, K.L.H.; Barber, L.; Murphy, J.; Cook, R. Social interaction contexts bias the perceived expressions of interactants. Emotion 2017, 17, 567-571. doi: 10.1037/emo0000257 32. Crivelli, C.; Russell, J.A.; Jarillo, S.; Fernández-Dols, J.-M. Recognizing spontaneous facial expressions of emotion in a small-scale society of Papua New Guinea. Emotion 2017, 17, 337-347. doi: 10.1037/emo0000236

33. Barrett, L.F.; Lindquist, K.A.; Gendron, M. Language as context for the perception of emotion. Trends Cogn. Sci. 2007, 11, 327-332. doi: 10.1016/j.tics.2007.06.003

34. Sowden, P.T.; Schyns, P.G. Channel surfing in the visual brain. Trends Cogn. Sci. 2006, 10, 538-545. doi: 10.1016/j.tics.2006.10.007

35. Fugate, J.M.B.; Gouzoules, H.; Barrett, L.F. Reading chimpanzee faces: evidence for the role of verbal labels in categorical perception of emotion. Emotion 2010, 10, 544-554. doi: 10.1037/a0019017

36. Nook, E.C.; Lindquist, K.A.; Zaki, J. A New look at emotion perception: concepts speed and shape facial emotion recognition. Emotion 2015, 15, 569-578. doi:

10.1037/a0039166

37. Halberstadt, J.B.; Niedenthal, P.M. Effects of emotion concepts on perceptual memory for emotional expressions. J. Pers. Soc. Psychol. 2001, 81, 587-598.

38. Krumhuber, E. G.; Hyniewska, S., \& Orlowska, A. Contextual effects on smile perception and recognition memory. Curr. Psychol. in press.

39. Goodenough, F.L.; Tinker, M.A. The relative potency of facial expression and verbal description of stimulus in the judgment of emotion. J. Comp. Psychol. 1931, 12, 365-370. doi: $10.1037 / \mathrm{h} 0071381$

40. Knudsen, H.R.; Muzekari, L.H. The effects of verbal statements of context on facial expressions of emotion. J. Nonverbal Behav. 1983, 7, 202-212.

41. Woll, S.B.; Martinez, J.M. The effects of biasing labels on recognition of facial expressions of emotion. Soc. Cogn. 1982, 1, 70-82. doi: 10.1521/soco.1982.1.1.70

42. Barsalou, L.W. Grounded cognition. Annu. Rev. Psychol. 2008, 59, 617-645. doi:

43. Robinson, M.D.; Clore, G.L. Belief and feeling: evidence for an accessibility model of emotional self-report. Psychol. Bull. 2002, 128, 934-960. doi:

44. Bourgeois, P.; Hess, U. The impact of social context on mimicry. Biol. Psychol. 2008, 77, 343-352. doi: 10.1037/0033-2909.128.6.934

45. Hess, U.; Kirouac, G. Emotion expression in groups. In Handbook of Emotion Lewis M.; Haviland-Jones J. Eds., Guilford Press: New York, 2000, 2nd Edition, pp. 368-381.

46. Coles, N.A.; Larsen, J.T.; Lench, H.C. A meta-analysis of the facial feedback literature: effects of facial feedback on emotional experience are small and variable. Psychol. Bull.

2019, 145, 610-651. doi: 10.1037/bul0000194

47. Wagenmakers, E.-J.; Beek, T.; Dijkhoff, L.; Gronau, Q.F.; Acosta, A.; Adams, R.B.; Albohn, D.N.; Allard, E.S.; Benning, S.D.; Blouin-Hudon, E.-M.; et al. Registered replication report: Strack, Martin, \& Stepper (1988). Perspect. Psychol. Sci. 2016, 11, 917928. doi: 10.1177/1745691616674458

48. Kätsyri, J.; de Gelder, B.; de Borst, A.W. Amygdala responds to direct gaze in real but not in computer-generated faces. Neuroimage 2020, 204, 116216. doi:

10.1016/j.neuroimage.2019.116216

49. Johnston, L.; Miles, L.; Neil Macrae, C. Why are you smiling at me? Social functions of enjoyment and non-enjoyment smiles. British Journal of Social Psychology 2010, 49, 107127. doi: 10.1348/014466609X412476 
50. Jospe, K.; Flöel, A.; Lavidor, M. The interaction between embodiment and empathy in facial expression recognition. Soc. Cogn. Affect. Neurosci. 2018, 13, 203-215. doi: 10.1093/scan/nsy005

51. Hsu, C.-T.; Sims, T.; Chakrabarti, B. How mimicry influences the neural correlates of reward: an fMRI study. Neuropsychologia 2018, 116, 61-67. doi:

10.1016/j.neuropsychologia.2017.08.018

52. Faul, F.; Erdfelder, E.; Lang, A.-G.; Buchner, A. G*Power 3: A flexible statistical power analysis program for the social, behavioral, and biomedical sciences. Behavior Research Methods 2007, 39, 175-191.

53. Martin, J.D.; Abercrombie, H.C.; Gilboa-Schechtman, E.; Niedenthal, P.M.

Functionally distinct smiles elicit different physiological responses in an evaluative context. Sci. Rep. 2018, 8, 3558. doi: 10.1038/s41598-018-21536-1

54. Wood, A.; Lupyan, G.; Sherrin, S.; Niedenthal, P. Altering sensorimotor feedback disrupts visual discrimination of facial expressions. Psychon. Bull. Rev. 2016, 23, 11501156. doi: 10.3758/s13423-015-0974-5

55. McLellan, T.; Johnston, L.; Dalrymple-Alford, J.; Porter, R. Sensitivity to genuine versus posed emotion specified in facial displays. Cognition Emotion 2010, 24, 1277-1292. doi: 10.1080/02699930903306181

56. Frank, M.G.; Ekman, P.; Friesen, W.V. Behavioral markers and recognizability of the smile of enjoyment. J. Pers. Soc. Psychol. 1993, 64, 83-93. doi: 10.1037/0022-3514.64.1.83 57. Baron-Cohen, S.; Wheelwright, S. The empathy quotient: an investigation of adults with asperger syndrome or high functioning autism, and normal sex differences. J. Autism Dev. Disord. 2004, 34, 163-175. doi: 10.1023/b:jadd.0000022607.19833.00 58. Krumhuber, E.G.; Kappas, A.; Manstead, A.S.R. Effects of dynamic aspects of facial expressions: a review. Emot. Rev. 2013, 5, 41-46. doi: 10.1177/1754073912451349 59. Tong, F.; Nakayama, K. Robust representations for faces: evidence from visual search. J. Exp. Psychol. Hum. Percept. Perform. 1999, 25, 1016-1035. doi: 10.1037//00961523.25.4.1016

60. Hugenberg, K.; Sacco, D.F. Social categorization and stereotyping: how social categorization biases person perception and face memory. Soc. Personal. Psychol. Compass 2008, 2, 1052-1072. doi: 10.1111/j.1751-9004.2008.00090.x

61. Corneille, O.; Huart, J.; Becquart, E.; Brédart, S. When memory shifts toward more typical category exemplars: accentuation effects in the recollection of ethnically ambiguous faces. J. Pers. Soc. Psychol. 2004, 86, 236-250. doi: 10.1037/0022-3514.86.2.236 62. Hugenberg, K.; Young, S.G.; Sacco, D.F. Social categorization influences face perception and face memory. In The Oxford Handbook of Face Perception; Rhoses, G., Calder, A., Johnson, M., Haxby, J.V., Eds.; Oxford University Press, 2011. 245-261. 63. Shaver, P.; Schwartz, J.; Kirson, D.; O'Connor, C. Emotion knowledge: further exploration of a prototype approach. Journal of Personality and Social Psychology 1987, 52, 1061-1086. doi: 10.1037//0022-3514.52.6.1061

64. Kirkham, A.J.; Hayes, A.E.; Pawling, R.; Tipper, S.P. Facial mimicry and emotion consistency: influences of memory and context. PLoS One 2015, 10, e0145731. doi: 10.1371/journal.pone.0145731

65. Sessa, P.; Schiano Lomoriello, A.; Luria, R. Neural measures of the causal role of observers' facial mimicry on visual working memory for facial expressions. Soc. Cogn. Affect. Neurosci. 2018, 13, 1281-1291. doi: 10.1093/scan/nsy095

Author Contributions: Conceptualization, A.O, M.R., P.S., and E.G.K.; methodology, A.O., M.R., and E.G.K.; software, A.O.; formal analysis, A.O. and E.G.K.; resources, E.G.K.; data curation, A.O., E.G.K; writing-original draft preparation, A.O.; writing-review and editing, A.O., M.R., and E.G.K.; visualization, A.O.; supervision, E.G.K. and P.S.; project administration, E.G.K.; funding 
acquisition, A.O, and P.S. All authors have read and agreed to the published version of the manuscript.

Funding: This research was supported by an internal grant for young scientists from the Institute of Psychology of Polish Academy of Sciences awarded to Anna Orlowska (IP PAN 2015 and IP PAN 2017).

Institutional Review Board Statement: The study was conducted according to the guidelines of the Declaration of Helsinki, and approved by the Ethics Committee of University College London (protocol code: EP2015_01; date of approval: 12 February 2015).

Informed Consent Statement: Informed consent was obtained from all subjects involved in the study.

Acknowledgments: The authors thank Inez Bawa, Weronika Kowalczyk, Iqra Malik, Luca Michael, Katarzyna Myszkowska, Sorelle Oates, Maya Rampal, Sabina Raus, Antonia Toneva for their help with data collection and Bronagh Allison for editing the manuscript.

Conflicts of Interest: The authors declare no conflict of interest. The funders had no role in the design of the study; in the collection, analyses, or interpretation of data; in the writing of the manuscript, or in the decision to publish the results. 